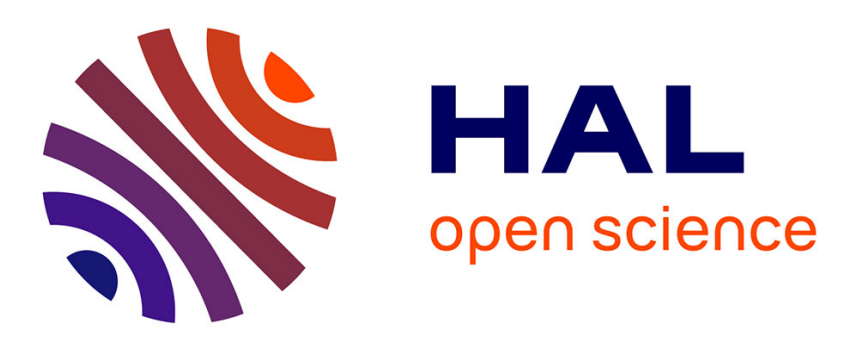

\title{
Generation of Atmospheric Turbulence with Unprecedentedly Large Reynolds Number in a Wind Tunnel
}

Lars Neuhaus, Michael Hölling, Wouter J. T. Bos, Joachim Peinke

\section{- To cite this version:}

Lars Neuhaus, Michael Hölling, Wouter J. T. Bos, Joachim Peinke. Generation of Atmospheric Turbulence with Unprecedentedly Large Reynolds Number in a Wind Tunnel. Physical Review Letters, 2020, 125 (15), pp.154503. 10.1103/PhysRevLett.125.154503 . hal-02964394

\section{HAL Id: hal-02964394 \\ https://hal.science/hal-02964394}

Submitted on 12 Oct 2020

HAL is a multi-disciplinary open access archive for the deposit and dissemination of scientific research documents, whether they are published or not. The documents may come from teaching and research institutions in France or abroad, or from public or private research centers.
L'archive ouverte pluridisciplinaire HAL, est destinée au dépôt et à la diffusion de documents scientifiques de niveau recherche, publiés ou non, émanant des établissements d'enseignement et de recherche français ou étrangers, des laboratoires publics ou privés. 


\title{
Generation of Atmospheric Turbulence with Unprecedentedly Large Reynolds Number in a Wind Tunnel
}

\author{
Lars Neuhaus $\odot,{ }^{1, *}$ Michael Hölling $\odot,{ }^{1}$ Wouter J. T. Bos $\odot,{ }^{2}$ and Joachim Peinke $\oplus^{1, \dagger}$ \\ ${ }^{1}$ Institute of Physics and ForWind, University of Oldenburg, 26129 Oldenburg, Germany \\ ${ }^{2}$ LMFA, CNRS, Ecole Centrale de Lyon, Université de Lyon, 69134 Ecully, France
}

(Received 22 April 2020; accepted 11 September 2020; published 9 October 2020)

\begin{abstract}
Generating laboratory flows resembling atmospheric turbulence is of prime importance to study the effect of wind fluctuations on objects such as buildings, vehicles, or wind turbines. A novel driving of an active grid following a stochastic process is used to generate velocity fluctuations with correlation lengths, and, thus, integral scales, much larger than the transverse dimension of the wind tunnel. The combined action of the active grid and a modulation of the fan speed allows one to generate a flow characterized by a four-decade inertial range and an integral scale Reynolds number of $2 \times 10^{7}$.
\end{abstract}

DOI: 10.1103/PhysRevLett.125.154503

Introduction.-Turbulence accompanies many aspects of everyday life. For instance, wind-induced turbulence distributes aerosols like seeds or fertilizing dust but can also cause extreme situations like typhoons or hurricanes. In the past decades, turbulent wind has gained special interest as a source of energy $[1,2]$.

The strength of turbulence is characterized by the Reynolds number

$$
\operatorname{Re}=\frac{U L}{\nu}
$$

where $U$ and $L$ are typical velocity and length scales, respectively, characterizing the large-scale structure of the flow, and $\nu$ is the kinematic viscosity of air. Taking a typical atmospheric wind speed of $10 \mathrm{~m} \mathrm{~s}^{-1}$ and a length $L>$ $1 \mathrm{~km}$ (the length scale on which the turbulence is created by shear) leads to values of $\mathrm{Re}$ of the order of $10^{9}$ and larger. This number determines the length and timescales for which turbulent fluctuations can be expected. Indeed, the smallest scale, where turbulent fluctuations can subsist in a fluid, are of the order of the Kolmogorov or dissipation length,

$$
\eta=\frac{L}{\operatorname{Re}^{3 / 4}},
$$

which shows that, for Reynolds numbers characterizing wind, the range of scales between the integral scale $L$ and dissipation length scale $\eta$ is very wide.

There is a special interest for these small scales, as the statistics of the fluctuations change fundamentally with decreasing scale. Whereas nearly Gaussian distributed fluctuations are characterizing the large scales, the smallscale fluctuations show heavy-tailed statistics, where extreme events with values of several standard deviations become much more likely than in a Gaussian field. Since atmospheric turbulence is driven on large scales larger than several kilometers, even objects as big as wind turbines or houses are exposed to such small-scale extreme events [3]. This challenges the desire to generate turbulent flow conditions, which resemble the atmospheric turbulence under laboratory conditions.

Controlled laboratory experiments of turbulent wind are most conveniently performed in wind tunnels, where the turbulent fluctuations are generated by blowing air through a grid. Such flows are restricted by several constraints. First, in order to avoid the influence of incompressibility, laboratory wind speeds should not exceed several tens of meters per second. Second, the spatial extent of the largescale velocity fluctuations which are generated at the grid is mostly of the order of one meter (a fraction of the diameter of the wind tunnel), corresponding to the length scale over which a velocity difference (shear) can be sustained. The biggest wind tunnels [4-6] allow one to increase the Reynolds number up to the order of millions, still well below the values of atmospheric turbulence. Finally, the third quantity determining $\operatorname{Re}$ is the kinematic viscosity, which can be lowered using different fluids, such as pressurized $\mathrm{SF}_{6}$ [7], cryogenic $\mathrm{N}_{2}$ [8], or low-temperature $\mathrm{He}$ [9-11]. However, the use of such fluids largely complicates the experimental installations.

An additional way to increase the Reynolds number is to act directly on the nature of the velocity fluctuations. One possibility to do so is to vary the shape of the grid [12]. Furthermore, instead of a grid with static grid bars, active grids can be used in order to enhance the intensity of the velocity fluctuations for a given mean wind speed [13]. A recent overview on active grids is given by Ref. [14]. Such grids allow one to increase the integral length scale compared to the mesh size of classical grids [13], but the large scales were found to be limited by the wind tunnel size $[15,16]$. The use of active grids introduces a new control parameter, corresponding to the temporal 
modulation of the grid bars allowing one to modify the properties of the generated turbulence [17].

In this Letter, an experimental approach is presented to generate a turbulent flow with an integral length scale much larger than the tunnel width, using a stochastic process for the active grid motion. The key idea is that, whereas the width of the tunnel is usually not larger than several meters, and the maximum transverse turbulent length scale is bounded by a fraction of this, the streamwise length of the tunnel may exceed this size so that larger correlation lengths can be observed in that direction. It is found that, using such long longitudinal correlation lengths, the obtained turbulence organizes itself in such a manner that it resembles a small-scale turbulence nested into a turbulent environment driven on very large scales. Intuitively, this corresponds to the case where a slice, of the size of the tunnel, is cut out of a much-larger-scale turbulence.

Experimental setup and turbulence excitation.Measurements are made in the closed test section of the Göttingen type wind tunnel in Oldenburg, having a cross section of $3 \times 3 \mathrm{~m}^{2}$ and a length of the measurement section of $30 \mathrm{~m}$ [18]. Wind velocities up to $42 \mathrm{~m} \mathrm{~s}^{-1}$ can be generated by four fans with a power of $110 \mathrm{~kW}$ each.

An active grid [18] with 80 rotating shafts and a mesh width of $M=0.143 \mathrm{~m}$ is mounted to the nozzle (Fig. 1). To the shafts rectangular flaps are mounted, which allow one to change the local blockage by their deflection angle. The shafts are separated in the middle of the grid and controlled individually by servo motors. A predefined motion protocol running on a real time controller defines the current shaft positions and can simultaneously modulate the wind tunnel fan speed and thereby the wind tunnel velocity.

To characterize the flow, an X-type hot wire 55P61 probe by Dantec Dynamics is placed on the center line of the closed test section. The hot-wire signals are sampled with a frequency of $20 \mathrm{kHz}$ after being low-pass filtered at $10 \mathrm{kHz}$. The measurements are repeated for different downstream positions from 1.43 to $20 \mathrm{~m}$ (which correspond to $10-140 \mathrm{M}$ ) using an in-house built $20 \mathrm{~m}$ traversing system.

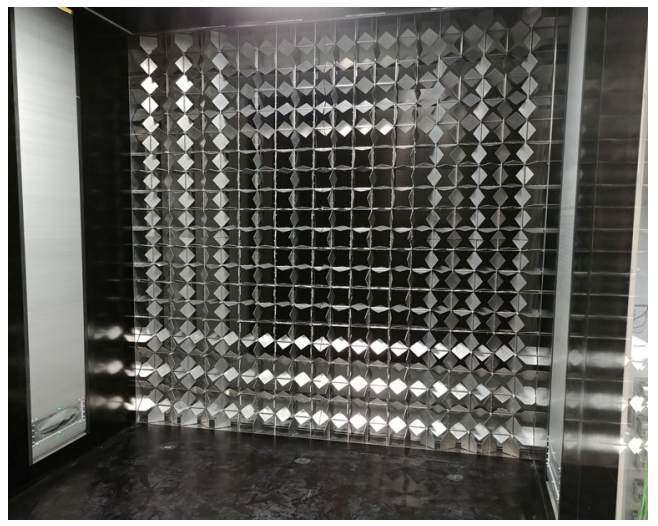

FIG. 1. Active grid installed in the closed test section.
In this Letter, a new approach to create atmosphericlike turbulence with large integral length scales is worked out. The idea is to use a stochastic process (e.g., OrnsteinUhlenbeck process), which exhibits a broadband decaying energy spectra on large scales, for the motion protocol of the active grid, defining the deflection angle of the flaps (see further details in Ref. [19]). Half the shafts are moving according to a predefined stochastic process, while the other half are following a motion to compensate the generated global blockage change. In consequence, the global blockage is kept constant, whereas the local blockage can vary strongly on short timescales. To ensure an undirected flow, neighboring shafts are pointing in opposite directions. Furthermore, the excitation can be expanded to even larger scales by a dynamic variation of the fan speed using the same type of stochastic process.

Analysis of the generated flow.-Two experimental approaches are presented. First, only the active grid is used for excitation, and the evolution of the turbulence downstream is discussed. Second, results of the additional manipulation of the flow by the wind tunnel fans are shown.

In the first approach, the fan speed is kept constant, generating a mean wind speed behind the active grid of roughly $\bar{u}=7 \mathrm{~m} \mathrm{~s}^{-1}$. Measurements are repeated for different downstream positions with the same 10 min lasting active grid motion protocol.

The aim is to generate very high Reynolds number turbulence with well-developed small and intermediate scales. Therefore, the downstream evolution of the power spectral density (PSD) of the velocity fluctuations is assessed (Fig. 2). Two frequencies $f_{\text {Flap }}:=\left(\bar{u} / L_{\text {Flap }}\right)$ and $f_{\mathrm{WT}}:=\left(\bar{u} / L_{\mathrm{WT}}\right)$ are defined, associated with the size of the flaps of the active grid $L_{\text {Flap }}=0.143 \mathrm{~m}$ and with the size of the wind tunnel $L_{\mathrm{WT}}=3 \mathrm{~m}$, respectively.

Close to the active grid (20M), the spectrum seems to be the superposition of two spectra, one containing energy in the highest frequencies $\left(f \geq f_{\text {Flap }}\right)$ and one containing energy in the lower frequencies $\left(f \leq f_{\mathrm{WT}}\right)$. The highfrequency part matches with the expected spectrum of the static grid (indicated by a colored dotted curve). In between ( $\left.f_{\mathrm{WT}} \leq f \leq f_{\text {Flap }}\right)$, some lack of energy (or gap) is found. A $-\frac{5}{3}$ power law exponent, as expected for fully developed turbulence [21], is observed only for the lowand high-frequency parts.

Farther downstream, the energy for the highest frequencies $(40 \mathrm{M})$ and low frequencies $(80 \mathrm{M})$ decreases, whereas the lack of energy in the medium frequencies is filled up by an energy cascade which extends now from the large scales, all the way down to the small scales. Even farther downstream $(90 \mathrm{M}$ and $100 \mathrm{M})$, the $-\frac{5}{3}$ power law spreads over a wide range of frequencies.

A constant slope from roughly $2 \times 10^{-1}$ to $3 \times 10^{2} \mathrm{~Hz}$ ( $\approx 3$ decades $)$ can be recognized for the far downstream position $(100 \mathrm{M})$. The gap between lower and higher 


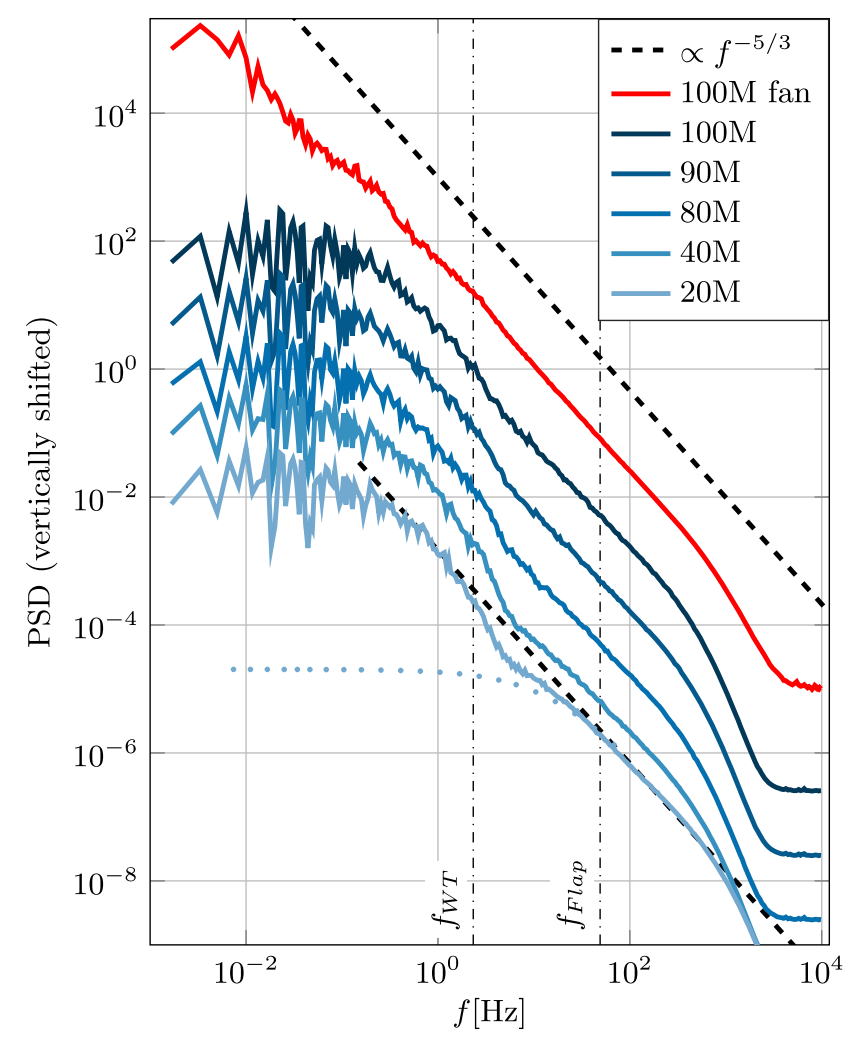

FIG. 2. Power spectral density (smoothed by exponentially growing bins) of generated turbulence at different downstream positions. For $20 \mathrm{M}$, the expected spectrum by the static grid is illustrated by a dotted curve. The statistical fluctuations at small frequencies are an artifact of the random signals that drive the grid (see further details in Ref. [19]); they are the same for all downstream positions.

frequencies is now closed, as its power spectra seem to have merged to form one large cascading process.

To quantify the range over which a constant exponent (close to $-\frac{5}{3}$ ) is found in the spectrum, a parameter $D$ (in units of decades $10^{D}$ ) is introduced. To do so, the spectrum is fitted by a polynomial in the frequency range of $10^{-1}$ to $5 \times 10^{3} \mathrm{~Hz}$. Regions in the spectra with almost no curvature are estimated by the second derivative of this fit. The extent $D$ of the power law region is determined by the part of the (smoothed) spectra where the second derivative is below a certain threshold (results do not depend sensitively on the choice of the threshold).

Close to the grid, $D$ decreases and stays close to a unity value until, at a downstream position of approximately 86M, $D$ rapidly increases (Fig. 3). After this rapid phasetransition-like behavior to a new developed turbulence state, $D$ increases further downstream until a value of 2.4 decades is observed at the farthest downstream positions (125-140 M).

Besides the power spectra, characterizing the variance of the fluctuations, intermittency is of particular interest. Therefore, the scale, or $\tau$, dependence of the probability density functions (PDF) of velocity increments

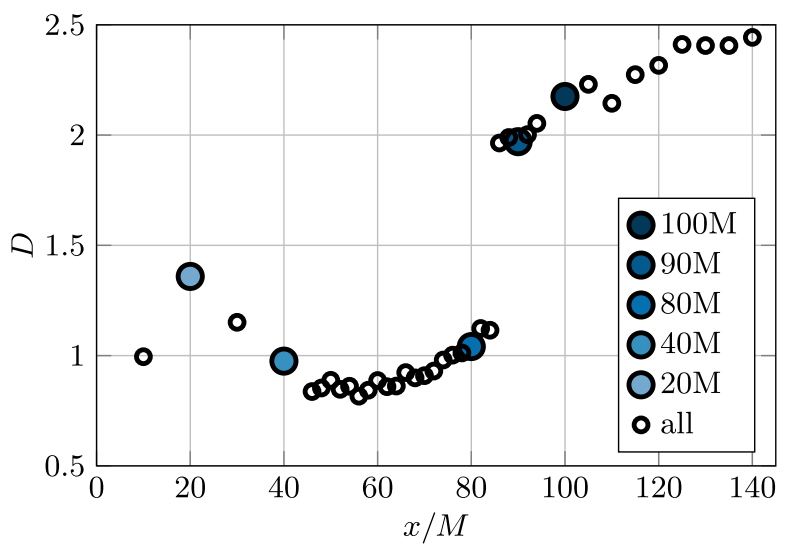

FIG. 3. Downstream development of the length $D$ of the range of scales over which a constant power law exponent is observed (in decades).

$u_{\tau}=u(t)-u(t+\tau)$ is investigated. The widths or the variances, $\left\langle u_{\tau}^{2}\right\rangle$, is directly connected to the PSD, as seen by the Wiener-Khinchin theorem [22]. To describe the shape of the PDFs at the timescale $\tau$, a shape factor

$$
\lambda^{2}=\frac{1}{4} \ln \left(\frac{\left\langle u_{\tau}^{4}\right\rangle}{3\left\langle u_{\tau}^{2}\right\rangle^{2}}\right)
$$

is analyzed [23], which is shown for different downstream positions in Fig. 4 in a semilog representation.

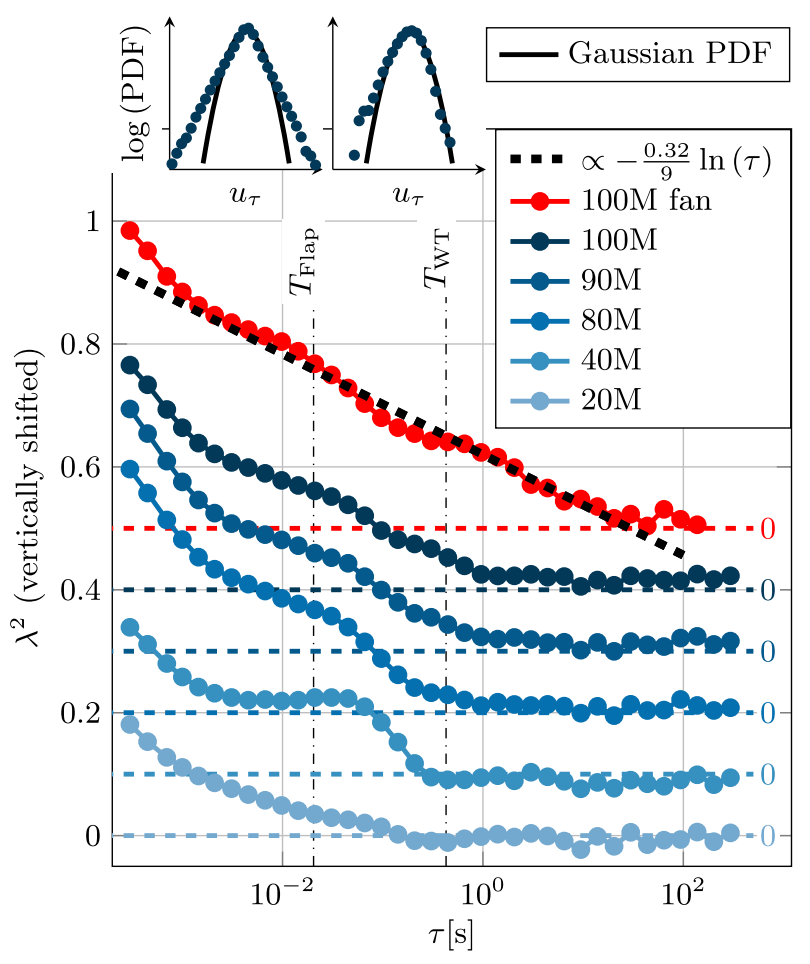

FIG. 4. Shape factor of generated turbulence at different downstream positions (colored dashed lines correspond to 0 , respectively). Increment PDFs are shown exemplarily for the scales of the flaps, $T_{\text {Flap }}$, and the wind tunnel, $T_{\mathrm{WT}}$, for the $100 \mathrm{M}$ position. 
On large scales, the PDFs of the velocity increments, $u_{\tau}$, are Gaussian and the shape factor is 0 . For smaller scales, the distributions become more heavy tailed, a phenomenon known as intermittency. This changing shape of the PDFs can be quantified by the increase of $\lambda^{2}$, which is connected to the increase of the dissipation rate fluctuations $\left\langle\left(\ln \epsilon_{r}\right)^{2}\right\rangle \propto \lambda^{2}(r)$. Following classic phenomenology [24], the turbulent cascade is characterized by a logarithmic increase of the shape factor

$$
\lambda^{2}(\tau)=\left[\Lambda_{0}^{2}-\mu \ln (\tau)\right] / 9 .
$$

with decreasing scales up to the largest intermittency value on small scales defined via $\Lambda_{0}$ [25]. The so-called intermittency factor $\mu$ can be estimated by the slope of $\lambda^{2}(\tau)$ or by the sixth-order structure function [26].

The closest upstream position (20M) shows a significant increase of $\lambda^{2}$ only for scales smaller than the flap size $T_{\text {Flap }}:=\left(L_{\text {flap }} / \bar{u}\right)$ (Fig. 4). Distributions close to Gaussian ones are found for $\tau \geq 0.1 \mathrm{~s}$. The logarithmic increase of the shape factor for smaller $\tau$ values shows where typical turbulent behavior is found, consistent with the results of the power spectrum.

Farther downstream (40M), the shape factor increases to small scales in two steps with a plateau close to $T_{\text {Flap }}$. The flow here seems to be dominated by two regions of different scales - one smaller than $T_{\text {Flap }}$ and one between $T_{\text {Flap }}$ and $T_{\mathrm{WT}}:=\left(L_{\mathrm{WT}} / \bar{u}\right)$. Farther downstream (80M), the two regions begin to merge. At the far downstream position $(100 \mathrm{M})$, the logarithmic law is extended over a larger range of scales, consistent with the findings of the extended scaling range in the PSD. Evaluating the slope in the semilog plot from $2 \times 10^{-3}$ to $1 \mathrm{~s}$ after Eq. (4), the value $\mu=0.258$ is obtained. This is in the expected range between 0.2 and 0.3 for ideal turbulence [26,27]. Consistent results are found by analysis of the sixth-order structure function.

In the second experimental approach, a low-frequency modulation of the wind tunnel fan speed by a stochastic process is added. The resulting mean wind speed $\bar{u}=8.4 \mathrm{~m} \mathrm{~s}^{-1}$ is slightly increased. Measurements are done for 100 min composed in ten independent 10 min intervals. The temperature was measured and found to be stable within $0.2 \mathrm{~K}$.

The PSD and the corresponding behavior of $\lambda^{2}$ for the far downstream (100M) position of this case, where both the fan and the active grid are exciting the flow, are also shown in Figs. 2 and 4 as upper red curves. It can be seen that more energy is fed into the system on larger scales and properties of ideal turbulence are extended over a wider range. This corresponds to an even higher Re turbulence state for which a $-\frac{5}{3}$ power law decay in the PSD over $\approx 4.5$ decades and a logarithmic increase of $\lambda^{2}$ over about four decades is found. The intermittency factor of $\mu=0.318$ is slightly increased.
Besides these statistical features of turbulence, the length scales are evaluated. The integral length scales are given by

$$
L_{i i}=\bar{u} \int_{0}^{\infty} R_{i i}(\tau) d \tau
$$

with $R_{i i}(\tau)$ the streamwise autocorrelation function of the $i$ component of the velocity fluctuations. The isotropic definition of the Taylor scale

$$
\lambda_{T}=\sqrt{\overline{u^{\prime 2}} / \overline{\left(\partial u^{\prime} / \partial x\right)^{2}}}
$$

is calculated by the velocity fluctuations $u^{\prime}$ in the streamwise $(x)$ direction. The length scale is numerically estimated for different time lags $\tau$ with the assumption of frozen turbulence and $\overline{\left(\partial u^{\prime} / \partial x\right)^{2}} \approx(\bar{u} \tau)^{-2}\left\langle u_{\tau}^{2}\right\rangle$ [28]. By a linear fit, $\lambda_{T}$ is extracted closed to $\tau=0$. These length scales are used to calculate the integral scale Reynolds number $\operatorname{Re}_{L}=\sqrt{\overline{u^{\prime 2}}} L / \nu$ and the Taylor Reynolds number $\operatorname{Re}_{\lambda_{T}}=\sqrt{\overline{u^{\prime 2}}} \lambda_{T} / \nu$.

For the case of active grid excitation, an integral length scale of $L_{11}=6 \mathrm{~m}$ and Reynolds numbers of $\mathrm{Re}_{L}=4.4 \times$ $10^{5}$ and $\operatorname{Re}_{\lambda_{T}}=3.230$ are achieved. Combined with the additional dynamic fan speed variation, an integral length scale of $L_{11}=120 \mathrm{~m}$ and Reynolds numbers of $\mathrm{Re}_{L}=$ $2.2 \times 10^{7}$ and $\operatorname{Re}_{\lambda_{T}}=12.800$ are estimated, comparable to atmospheric flows with values of about 10.000 [29]. The integral length scales of the transversal component are found to be $L_{22}=0.185 \mathrm{~m}$ (active grid) and $L_{22}=$ $0.505 \mathrm{~m}$ (active grid and fan excitation) due to the geometry of the wind tunnel. Most interestingly, the intermittency of velocity increments measured by the shape factor $\lambda^{2}$ behaves as if $L_{22}$ is of the size of $L_{11}$ as is shown in Fig. S3 in Supplemental Material [19].

Conclusion.-The new approach of excitation of a turbulent flow on scales larger than the experimental setup itself seems to drastically increase the attainable Reynolds number in wind tunnel turbulence. The investigation of such flows needs long wind tunnels. In the present setup, measurements $80 \mathrm{M}$ behind the grid showed the merger of the spectral ranges associated with small- and large-scale excitation, respectively. At positions $x \geq 100 \mathrm{M}$ behind the grid, a continuous scaling range of more than four decades and an integral length scale of more than $100 \mathrm{~m}$ were measured.

The analysis of the transverse velocity component is showing comparable results like the logarithmic behavior of the shape factors for timescales up to $0.5 \mathrm{~s}$, indicating approximate isotropy in the small scales (for further discussion see [19]).

It is puzzling that the generated flow shows, at the small scales, characteristics of turbulence with scales larger than the width of the wind tunnel. A first answer is obtained by realizing that the energy fluctuations are generated 
differently in the present case. Whereas in classic grids a transverse shear is generated by the grid bars, in the present case the active grid and wind tunnel fans add a lowfrequency longitudinal shear pattern to this. This allows one to inject energy into the turbulence at longitudinal scales beyond the tunnel width (for further discussion, see [19]).

The presence of energy at these low frequencies thereby may act, through nonlocal triadic interaction, as a catalyst for the nonlinear activity at much smaller scales [30-32]. The rapid filling of the spectral gap, observed in Fig. 2, seems to be a direct consequence of this. The lowfrequency excitation is therefore not purely decorative but allows one to attain turbulence over a wider range of scales than normal, high-frequency excitation alone would allow one to attain. In our opinion, it is important that this is confirmed by the shape factor which shows a large scaling range, reminiscent of very large Reynolds number turbulence.

Thereby, the present investigation allows one to investigate very high Reynolds number turbulence, with small scales which are still relatively large, enabling the experimental study of the interaction of small-scale high Reynolds number turbulence in the laboratory. This opens interesting new rescaling applications for experimental investigations of the impact of high Re turbulence on objects, like the impact of turbulent wind on wind turbines.

We thank the Federal Ministry for Economic Affairs and Energy of Germany (BMWi) + MWK Lower Saxony, DFG (Grossgeraet) for funding parts of this work. Furthermore, we thank Abdulkarim Abdulrazek, André Fuchs, Nils Haverland, Pascal Knebel, Lars Kröger, Ingrid Neunaber, Jaroslaw Puczylowski, Piyush Singh, and Matthias Wächter for fruitful discussions.

*lars.neuhaus@uol.de †joachim.peinke@uol.de

[1] C. Meneveau, Big wind power: seven questions for turbulence research, J. Turbul. 20, 2 (2019).

[2] P. Veers, K. Dykes, E. Lantz, S. Barth, C. L. Bottasso, O. Carlson, A. Clifton, J. Green, P. Green, H. Holttinen, D. Laird, J. K. Lundquist, C. Meneveau, L. Pao, J. Peinke et al., Grand challenges in the science of wind energy, Science 366, eaau2027 (2019).

[3] F. Böttcher, S. Barth, and J. Peinke, Small and large scale fluctuations in atmospheric wind speeds, Stochastic Environ. Res. Risk Assess. 21, 299 (2007).

[4] S. G. Saddoughi and S. V. Veeravalli, Local isotropy in turbulent boundary layers at high Reynolds number, J. Fluid Mech. 268, 333 (1994).

[5] M. Bourgoin, C. Baudet, S. Kharche, N. Mordant, T. Vandenberghe, S. Sumbekova, N. Stelzenmuller, A. Aliseda, M. Gibert, P.-E. Roche, E. Bodenschatz, G. Bewley, M. Sinhuber, Örlü, J. Puczylowski, J. Peinke et al., Investigation of the small-scale statistics of turbulence in the
Modane S1MA wind tunnel, CEAS Aeronaut. J. 9, 269 (2018).

[6] DNW German-Dutch wind tunnels, large low-speed facility, https://www.dnw.aero/wind-tunnels/llf (8 april 2020).

[7] E. Bodenschatz, G. P. Bewley, H. Nobach, M. Sinhuber, and H. Xu, Variable density turbulence tunnel facility, Rev. Sci. Instrum. 85, 093908 (2014).

[8] DNW German-Dutch wind tunnels, Kryo-Kanal Köln, https://www.dnw.aero/wind-tunnels/kkk/ (23 september, 2020).

[9] F. Heslot, B. Castaing, and A. Libchaber, Transitions to turbulence in helium gas, Phys. Rev. A 36, 5870 (1987).

[10] J. Niemela and K. Sreenivasan, The use of cryogenic helium for classical turbulence: promises and hurdles, J. Low Temp. Phys. 143, 163 (2006).

[11] S. Pietropinto, C. Poulain, C. Baudet, B. Castaing, B. Chabaud, Y. Gagne, B. Hébral, Y. Ladam, P. Lebrun, O. Pirotte, and P. Roche, Superconducting instrumentation for high Reynolds turbulence experiments with low temperature gaseous helium, Physica (Amsterdam) 386C, 512 (2003).

[12] D. Hurst and J. Vassilicos, Scalings and decay of fractalgenerated turbulence, Phys. Fluids 19, 035103 (2007).

[13] H. Makita, Realization of a large-scale turbulence field in a small wind tunnel, Fluid Dyn. Res. 8, 53 (1991).

[14] L. Mydlarski, A turbulent quarter century of active grids: from Makita (1991) to the present, Fluid Dyn. Res. 49, 061401 (2017).

[15] C. Küchler, G. Bewley, and E. Bodenschatz, Experimental study of the bottleneck in fully developed turbulence, J. Stat. Phys. 175, 617 (2019).

[16] K. P. Griffin, N. J. Wei, E. Bodenschatz, and G. P. Bewley, Control of long-range correlations in turbulence, Exp. Fluids 60, 55 (2019).

[17] H. E. Cekli, R. Joosten, and W. van de Water, Stirring turbulence with turbulence, Phys. Fluids 27, 125107 (2015).

[18] L. Kröger, J. Frederik, J.-W. van Wingerden, J. Peinke, and M. Hölling, Generation of user defined turbulent inflow conditions by an active grid for validation experiments, J. Phys. Conf. Ser. 1037, 052002 (2018).

[19] See Supplemental Material at http://link.aps.org/ supplemental/10.1103/PhysRevLett.125.154503 for details on the excitation, isotropy, homogeneity, and kinetic energy balance for flow generated by an active grid, which includes Ref. [20].

[20] C. Klipp, Turbulence anisotropy in the near-surface atmosphere and the evaluation of multiple outer length scales, Boundary-layer Meteorol. 151, 57 (2014).

[21] A. N. Kolmogorov, The local structure of turbulence in incompressible viscous fluid for very large Reynolds numbers, C. R. Acad. Sci. URSS 30, 299 (1941).

[22] U. Frisch, Turbulence: The Legacy of AN Kolmogorov (Cambridge University Press, Cambridge, England, 1995).

[23] F. Chilla, J. Peinke, and B. Castaing, Multiplicative process in turbulent velocity statistics: A simplified analysis, J. Phys. II 6, 455 (1996).

[24] A. N. Kolmogorov, A refinement of previous hypotheses concerning the local structure of turbulence in a viscous incompressible fluid at high Reynolds number, J. Fluid Mech. 13, 82 (1962). 
[25] B. Castaing, Y. Gagne, and E. Hopfinger, Velocity probability density functions of high Reynolds number turbulence, Physica (Amsterdam) 46D, 177 (1990).

[26] A. Arneodo, C. Baudet, F. Belin, R. Benzi, B. Castaing, B. Chabaud, R. Chavarria, S. Ciliberto, R. Camussi, F. Chilla, B. Dubrull, Y. Gagne, B. Hebral, J. Peinke, W. van de Water et al., Structure functions in turbulence, in various flow configurations, at Reynolds number between 30 and 5000, using extended self-similarity, Europhys. Lett. 34, 411 (1996).

[27] P. Davidson, Turbulence: An Introduction for Scientists and Engineers (Oxford University Press, Oxford, 2004).

[28] D. Aronson and L. Löfdahl, The plane wake of a cylinder: Measurements and inferences on turbulence modeling, Phys. Fluids A 5, 1433 (1993).
[29] E. F. Bradley, R. Antonia, and A. Chambers, Turbulence Reynolds number and the turbulent kinetic energy balance in the atmospheric surface layer, Boundary-Layer Meteorol. 21, 183 (1981).

[30] J. A. Domaradzki and R. S. Rogallo, Local energy transfer and nonlocal interactions in homogeneous, isotropic turbulence, Phys. Fluids A 2, 413 (1990).

[31] F. Waleffe, The nature of triad interactions in homogeneous turbulence, Phys. Fluids A 4, 350 (1992).

[32] A. Alexakis, P. D. Mininni, and A. Pouquet, Imprint of Large-Scale Flows on Turbulence, Phys. Rev. Lett. 95, 264503 (2005). 